\title{
Frecuencia de Anaplasma sp. y Ehrlichia sp. en caninos con sintomatología compatible con enfermedad hemoparasitaria en Huánuco, Perú
}

Frequency of Anaplasma sp. and Ehrlichia sp. in dogs with symptoms compatible with hemoparasitic disease in Huánuco, Perú

\author{
Walter Richard Tasayco Alcántara ${ }^{1}$, Juan Marco Vásquez Ampuero ${ }^{1}$
}

\section{RESUMEN}

El estudio tuvo como objetivo determinar la frecuencia de Anaplasma sp. y Ehrlichia sp. en caninos domésticos con signos clínicos compatibles con enfermedad hemoparasitaria de centros veterinarios de los distritos de Huánuco, Amarilis y Pilcomarca. Para ello se seleccionó en forma intencional a 100 perros, a quienes se extrajo muestras de sangre para realizar análisis hematológico y una prueba inmunocromatográfica doble para detección de anticuerpos de Anaplasma sp. y Ehrlichia sp.; además de aplicar un cuestionario epidemiológico a los propietarios. El estudio se realizó entre los meses de mayo y octubre del año 2020. El 61\% fue positivo a Anaplasma, el 85\% a Ehrlichia, y en el 55\% (55/100) de los perros existió coinfección con ambos patógenos. El hemograma de los perros positivos a Ehrlichia sp. y Anaplasma sp., presentaron anemia, leucopenia y trombocitopenia como los signos clínicos mas frecuentes. Se encontró asociación entre los resultados positivos a Ehrlichia y la variable raza, trombocitopenia y el signo clínico de mucosas pálidas en los perros $(\mathrm{p}<0,05)$. Los hallazgos encontrados a partir de este estudio muestran una alta frecuencia de Ehrlichia sp., y se confirma por primera vez la coinfección de Anaplasma sp. + Ehrlichia sp., en Huánuco.

PALABRAS CLAVE: Anaplasmosis, erliquiosis, vectores, zoonosis, salud pública.

\section{SUMMARY}

The study aimed to determine frequency of Anaplasma sp. y Ehrlichia sp. in domestic canines with compatible symptoms with hemoparasitic disease of veterinary centers of the Huánuco, Amarilis y Pilcomarca districts. For this, it was selected intentionally to 100 dogs, from whom blood samples are drawn to perform haematological analysis and double immunochromatographic test for antibody detection of Anaplasma and Ehrlichia; in addition to applying a epidemiological questionnaire to the owners. The study was conducted between the months of may and october 2020 . The $61 \%$ was positive to Anaplasma, the $85 \%$ to Ehrlichia, and in the $55 \%(55 / 100)$ of the dogs existed coinfection with both pathogens. The haemogram of the positive dogs Anaplasma y Ehrlichia presented anemia, leucopenia and thrombocytopenia like the most frequent clinical signs. Association found between the positive results to Ehrlichia and the race variable, thrombocytopenia, and the clinic sign of pale mucous membranes in the dogs $(\mathrm{p}<0.05)$. The findings found from this study show a high frequency of Ehrlichia sp., is confirmed for the first time coinfection Anaplasma sp. + Ehrlichia sp. in Huánuco.

KEY WORDS: Anaplasmosis, ehrlichiosis, vector, zoonoses, public health.

Facultad de Medicina Veterinaria y Zootecnia, Universidad Nacional Hermilio Valdizán. Huánuco Péru. 


\section{INTRODUCCIÓN}

Las enfermedades trasmitidas por vectores que afectan a caninos y felinos (VBDs), son causadas por un amplio rango de patógenos que comprenden virus, bacterias, protozoos y helmintos, trasmitidos a perros y gatos por diferentes especies de vectores artrópodos. Algunos de estos pueden representar una seria amenaza para la salud y el bienestar animal, y constituye un desafío diagnóstico para los médicos veterinarios, debido al amplio espectro de manifestaciones clínicas, los largos períodos prepatentes y la frecuente ocurrencia de co-infecciones (Alho et al., 2017).

Ejemplo de VBDs son las infecciones por ehrlichiosis y anaplasmosis. Ehrlichia canis es una bacteria gram negativa, obligada intracelular, que infecta los monocitos y es el agente causal primario de la ehrlichiosis monocítica canina (Greene, 2008). Riphicephalus sanguineus trasmite $E$. canis a los perros, tanto transestadialmente como intraestadialmente (Stich et al., 2008). A su vez Anaplasma platys es una bacteria intracelular obligatoria gram negativa que infecta a las plaquetas ocasionando trombocitopenia cíclica canina. El perro es el huésped reservorio primario para $A$. platys, y hasta la fecha no ha demostrado infectar a los seres humanos. Tambien es trasmitida por el Riphicephalus sanguineus (Simpson et al., 1991).

Las infecciones por A. platys generalmente se encuentran en las mismas regiones geográficas donde se reporta E. canis, y frecuentemente se detectan evidencias de exposición o infección con ambos microorganismos en el mismo perro (Ybañez et al., 2012; Harrus et al., 1997). Ambos patógenos se encuentran en todos los continentes del mundo, pero son más prevalentes en climas tropicales y subtropicales (Gaunt et al, 2010). Una serie de estudios han demostrado la presencia de estas coeinfecciones en diferentes países como Qatar (Alho et al., 2017), sudeste de China (Zhang et al., 2017), en la ciudad de Araguaína, estado de Tocantins, Brasil (Barbosa et al., 2018) y en la región Norte del estado de Paraná (Ferreira da Silva et al., 2012), entre otros países.

En Huánuco existe una población canina que incluye a perros de casa y callejeros infestados con garrapatas. Asimismo, los profesionales veterinarios reportan perros con signos clínicos de enfermedades trasmitidas por garrapatas, como fiebre, decaimiento, anemia, leucopenia y trombocitopenia; y que luego de realizar pruebas de laboratorio como observación de frotis sanguíneo y test rápido, resultaron positivos a ehrlichiosis (Huerto y Dámaso, 2015; Tasayco et al., 2017). Sin embargo, se sospecha de la presencia de otros patógenos trasmitidos por garrapatas como Anaplasma, Babesia, Dirofilaria y Hepatozoon; de los cuales no se tiene información por la ausencia de estudios rigurosos. En este contexto, el objetivo del estudio fue conocer la frecuencia, valores hematológicos y los factores asociados a la infección por Anaplasma y Ehrlichia en perros con signos compatibles de estas enfermedades, atendidos en consultorios veterinarios de tres distritos de la provincia de Huánuco, Perú.

\section{MATERIAL Y MÉTODOS}

El estudio se desarrolló en la provincia de Huánuco que se ubica a 1,800 m.s.n.m., en el valle formado por el río Huallaga, ubicado en la Región Centro Oriental del país. El clima es seco y soleado, con una temperatura promedio de $24^{\circ} \mathrm{C}$ durante todo el año. La investigación corresponde a un estudio transversal analítico. La población a estudiar, fueron los caninos de los distritos de Huánuco, Amarilis y Pilcomarca, las ciudades más pobladas de la Provincia de Huánuco que en conjunto reunía alrededor de 196,627 habitantes (Instituto Nacional de Estadística e Informática, 2017). Una aproximación al calculo de la muestra fue realizado mediante la formula de comprobación de una proporción para poblaciones desconocidas (Daniel, 2002) utilizando las siguientes restricciones: proporción referencial del 50\% (valor utilizado cuando se desconoce una proporción referencial) un nivel de confianza del $95 \%$ y un error máximo admisible del 10\%. El tamaño de muestra calculado fue de 96 canes con sintomatología compatible con enfermedad homoparasitaria.

Se logró la participación voluntaria de tres Centros Veterinarios; uno en cada ciudad (Huánuco, Amarilis y Pilcomarca). Los criterios de inclusión fueron: perros que presentaban cualquiera de los siguientes signos compatible de enfermedad consideradas en el estudio (fiebre, mucosas pálidas, letargo, anorexia, hemorragia, signos neurológicos, ictericia, queratitis), de ambos sexos, mayores de 2 meses de edad e infestados con garrapatas o con antecedentes de haberlas tenido en el mes previo. Los criterios de exclusión fueron: perros agresivos que no pudieran ser controlados, por el riesgo que representaban para la persona que tomaba la muestra, dueños de perros 
que no quieran que sus mascotas participen, perros enfermos que se encontraban en tratamiento por alguna otra enfermedad y hembras gestantes.

Se elaboró una ficha epiemiológica que incluyo la siguiente información: edad ( $<1, \geq 1 \mathrm{y}<5, \geq 5$ años), sexo (macho o hembra), distrito de procedencia (Amarilis, Pilcomarca o Huánuco), tipo de pelaje (corto o largo), acceso a la calle (si o no), infestación por garrapatas en el último mes (si o no) y presencia de síntoma compatibles con enfermedad hemoparasitaria (fiebre, anorexia, letargo, mucosas pálidas, ictericia, otros o ninguno). La ficha incluyó tambien una sección de registro de los resultados del hemograma (valores del examen hematológico correspondientes a la serie blanca y la serie roja)

Después de identificar y anotar los datos de la reseña del animal, antecedentes y signos clínicos, y datos del propietario; se procedió a la toma de muestra de sangre con el consentimiento del propietario. Tras una adecuada sujeción, se procedió a rasurar la zona del miembro anterior donde se puedo visualizar la vena cefálica, luego se desinfectó el área con alcohol, para extraer la muestra de sangre usando una aguja de calibre proporcional al tamaño del animal. Se recolectaron aproximadamente $2 \mathrm{ml}$ de sangre de la vena cefálica utilizando el sistema al vacio y tubos con EDTA.

El diagnóstico serológico se realizó mediante el ensayo inmunocromatográfico doble en fase sólida para detección de anticuerpos de Anaplasma sp. y Ehrlichia sp. en suero, plasma o sangre entera, específico para que tenía una sensibilidad y especificidad del 97\% (Waner et al., 2001). El Kit de prueba (Jiangsu Fusida Biotechnology Co., Ltd), es un inmunoensayo cromatográfico para la detección cualitativa de anticuerpos de Anaplasma sp., y Ehrlichia sp., en suero, plasma o sangre total canina. Tiene las letras " $T$ " y " $C$ " que indican la aparición de franjas de prueba y de control respectivamente en la bandeja de prueba. La franja o línea de control de color rojo púrpura es visible o aparece siempre cuando el test funciona correctamente, y la franja de prueba púrpura aparecerá en la bandeja, en cada uno de las pozas de Anaplasma sp., y Ehrlichia sp., cuando la muestra presenta anticuerpos contra dichos agentes etiológicos, respectivamente (Kelly et al., 2013).

La presencia única de una franja "C" en el pozo tanto de Anaplasma sp., y Ehrlichia sp., indica un resultado negativo. La presencia de dos franjas de color púrpura en " $\mathrm{C}$ " y " $\mathrm{T}$ ", en cada poza, tanto de Anaplasma sp., y Ehrlichia sp., sin importar cual banda aparece primero, indica un resultado positivo. Si no aparece la franja de color rojo púrpura en "C", en ambas pozas, después de realizar la prueba, el resultado se considera inválido.

La otra parte de la muestra de sangre entera se trasladó a una Clínica Veterinaria privada para realizar el hemograma en un equipo de análisis hematológico automatizado y calibrado AUTO HEMATOLOGY ANALYZER KT-6300 VET Genrui ${ }^{\circledR}$. El referido equipo expresa resultados sobre Leucocitos y recuento diferencial, Eritrocitos, Hemoglobina, Hematocrito, VCM, HCM, CHCM, y Plaquetas.

Los resultados de las pruebas diagnosticas a Anaplasma sp., y Ehrlichia sp., se resumieron mediante estadística descriptiva utilizando frecuencias absolutas y relativas, de acuerdo a las categorías descritas para las variables independientes consideradas en el estudio. La asociación entre las variables raza, sexo, antecedente de garrapatas, presencia de signos clínicos y garrapatas al examen clínico y los animales positivos a la prueba de detección de anticuerpos frente a Anaplasma sp. y Ehrlichia sp. fue evaluada mediante la prueba de Chi Cuadrado considerando un valor de significancia de 0.05 .

Los resultados del análisis hematológico fueron resumidos mediante estadística descriptiva utilizando la media como medida de tendencia central y la desviación estándar como medida de dispersión, para los datos agrupados de acuerdo al resultado positivo o negativo y clasificados según resultado a las pruebas diagnosticas a Anaplasma sp., y Ehrlichia sp. La comparación de las medias entre los grupos se realizo mediante la prueba de T de Student de independencia. El estudio tomó en cuenta todos los aspectos que garantizaban el bienestar del animal, principalmente durante la revisión del mismo y en el momento de la toma de muestra de sangre. La participación en el estudio fue confirmado mediante la firma de un consentimiento informado por parte del responsable del animal.

\section{RESULTADOS}

El estudio recuperó muestras de 100 perros obtenidos en consultorios veterinarios de los distritos de Pilcomarca, Amarilis y Huánuco. Predominaron animales de raza mestiza, sexo macho, de edad adulta entre 1 a 6 años, pelo corto y que predominantemente 


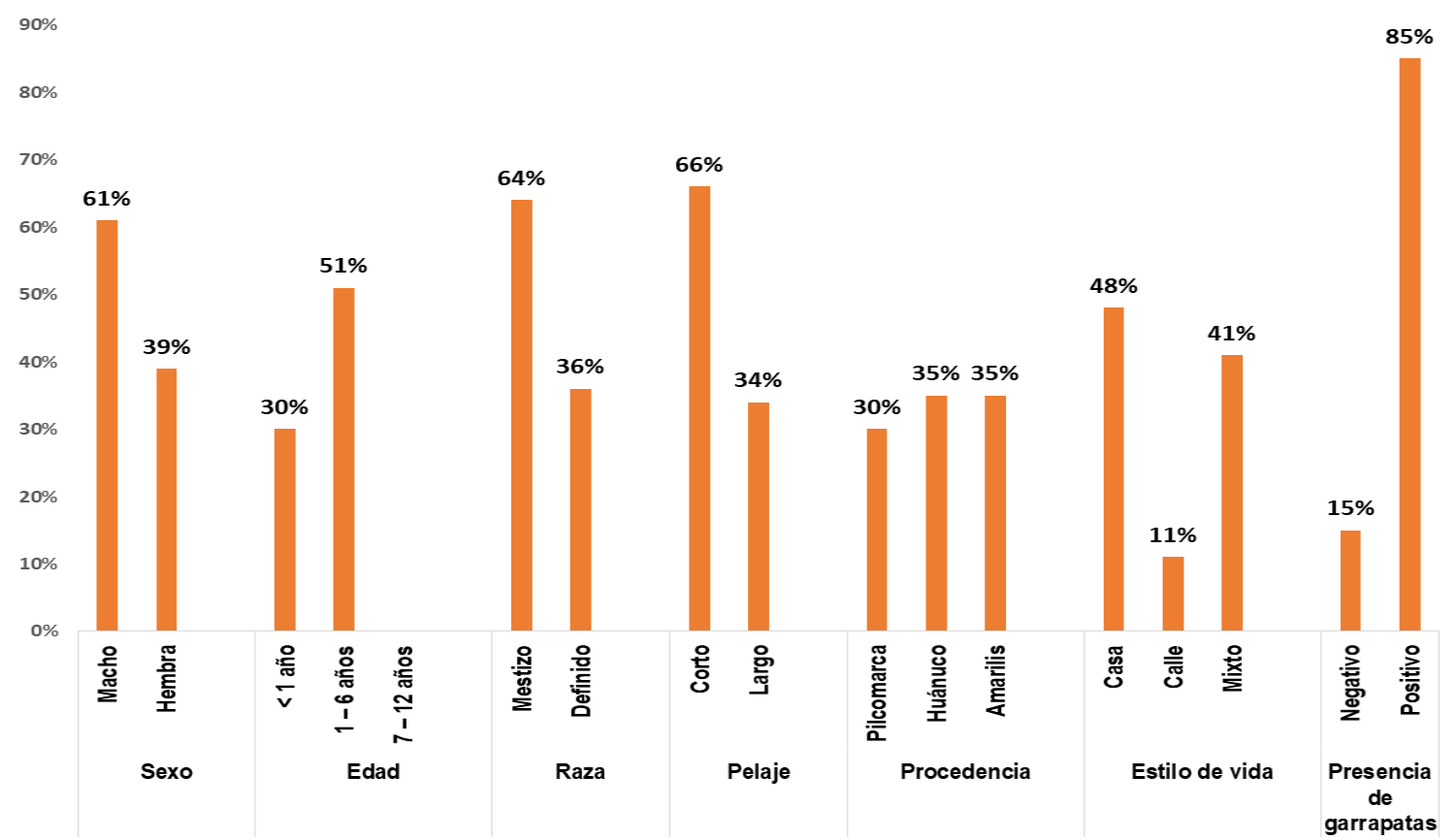

Gráfico 1. Caracteristicas de los perros participantes del estudio. Huánuco 2020.

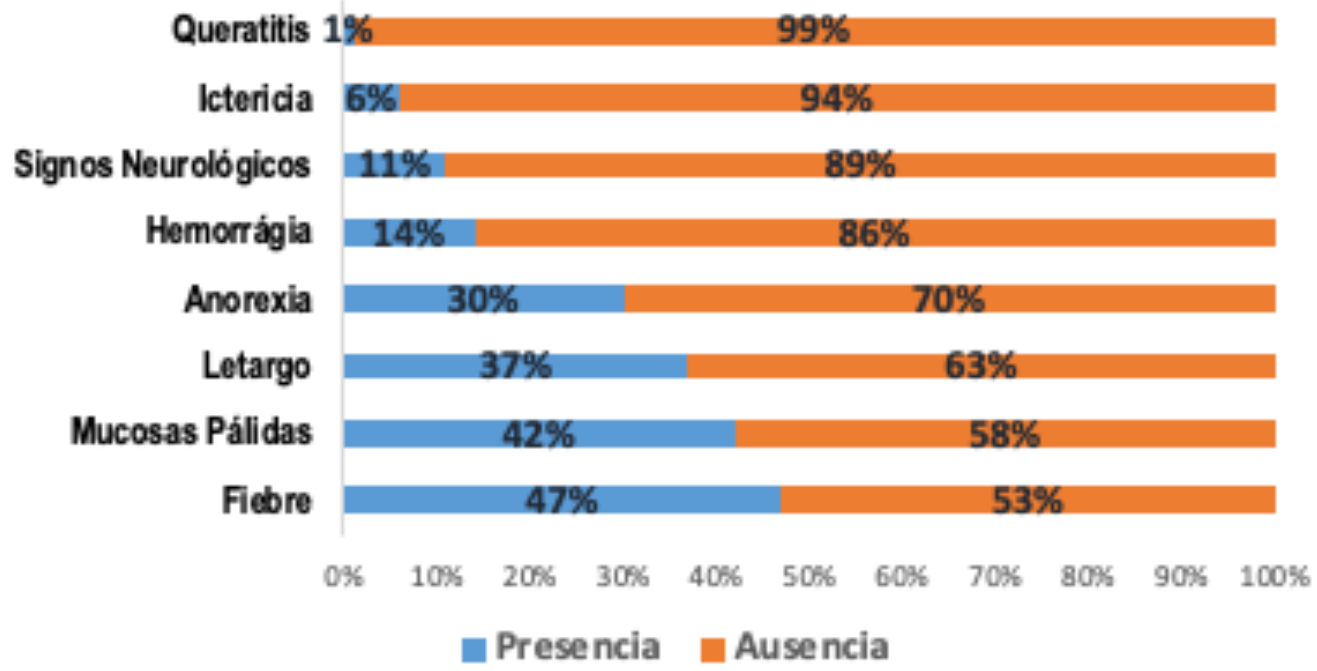

Gráfico 2. Signos clínicos compatibles con enfermedades hemoparasitarias presentados entre los animales incluidos en el estudio.

vivian en casa y con presencia de garrapatas. La distribución de las características de los animales se presenta en el gráfico 1. En el gráfico 2 se muestra la frecuencia de los signos clínicos presentes en los animales en estudio, siendo las más comunes: fiebre, mucosas pálidas, letargo y anorexia.

De los 100 perros estudiados, 30\% fueron exclusivamente positivos a Ehrlichia sp., 6\% exclusivamente positivos a Anaplasma Sp., y 55\% fueron positivos a ambos agentes etiológicos. (Ehrlichia Sp. + Anaplasma Sp.). Solo se encontró asociación entre la variable raza y los resultados positivos a la prueba diagnóstica. La distribución de los resultados positivos según características demográficas estudiadas se presenta en la tabla 1 .

En relación a la presencia de resultados positivos a Ehrlichia sp. y Anaplasma sp. y la presentación de signos clínicos, la prueba de Chi Cuadrado 
Tabla 1. Distribución de los resultados positivos a Ehrilichia sp. y Anaplama sp. según características demográficas de los perros estudiados. Huánuco - Perú, 2020.

\begin{tabular}{|c|c|c|c|c|c|c|c|}
\hline \multirow{2}{*}{ Categorías } & \multirow{2}{*}{ Total } & \multicolumn{3}{|c|}{ Ehrilicha Sp. (+) } & \multicolumn{3}{|c|}{ Anaplasma Sp. (+) } \\
\hline & & n. & $\%$ & Sig. & $\mathrm{n}$ & $\%$ & Sig. \\
\hline \multicolumn{8}{|l|}{ Raza } \\
\hline Mestizo & 64 & 51 & 79,7 & \multirow{2}{*}{0,047} & 39 & 60,9 & \multirow{2}{*}{0,986} \\
\hline Definido & 36 & 34 & 94,4 & & 22 & 61,1 & \\
\hline \multicolumn{8}{|l|}{ Sexo } \\
\hline Macho & 61 & 54 & 88,5 & \multirow{2}{*}{0,217} & 40 & 65,6 & \multirow{2}{*}{0,241} \\
\hline Hembra & 39 & 31 & 79,5 & & 21 & 53,8 & \\
\hline \multicolumn{8}{|l|}{ Pelaje } \\
\hline Corto & 66 & 54 & 81,8 & \multirow{2}{*}{0,214} & 39 & 59,1 & \multirow{2}{*}{0,586} \\
\hline Largo & 34 & 31 & 91,2 & & 22 & 64,7 & \\
\hline \multicolumn{8}{|l|}{ Estilo de vida } \\
\hline Casa & 48 & 39 & 81,3 & \multirow{3}{*}{0,582} & 31 & 64,6 & \multirow{3}{*}{0,703} \\
\hline Calle & 11 & 10 & 90,9 & & 7 & 63,6 & \\
\hline Mixto & 41 & 36 & 87,8 & & 23 & 56,1 & \\
\hline \multicolumn{8}{|c|}{ Presencia de garrapatas } \\
\hline Negativo & 15 & 12 & 80,0 & \multirow{2}{*}{0,556} & 7 & 46,7 & \multirow{2}{*}{0,217} \\
\hline Positivo & 85 & 73 & 85,9 & & 54 & 63,5 & \\
\hline \multicolumn{8}{|l|}{ Procedencia } \\
\hline Pilcomarca & 30 & 28 & 93,3 & \multirow{3}{*}{0,294} & 23 & 76,7 & \multirow{3}{*}{0,097} \\
\hline Huánuco & 35 & 28 & 80,0 & & 20 & 57,1 & \\
\hline Amarilis & 35 & 29 & 82,9 & & 18 & 51,4 & \\
\hline \multicolumn{8}{|l|}{ Edad } \\
\hline$<1$ año & 30 & 27 & 90,0 & \multirow{3}{*}{0,420} & 22 & 73,3 & \multirow{3}{*}{0,188} \\
\hline $1-6$ años & 51 & 41 & 80,4 & & 27 & 52,9 & \\
\hline 7 - 12 años & 19 & 17 & 89,5 & & 12 & 63,2 & \\
\hline
\end{tabular}

Sig. $<0.05$ indica que las proporciones son significativamente diferentes.

confirma que no existia asociación entre estos agentes etiológicos y los signos de fiebre, anorexia, letargia, ictericia, hemorragia, signos neurológicos y queratitis. Sin embargo, el signo clínico de mucosas pálidas sí tuvo asociación significativa con Ehrlichia sp., pero no con Anaplasma sp. Los resultados en detalle se presentan en la tabla 2.

La evaluación de los valores hematológicos obtenidos en los perros con resultados positivos y negativos a Ehrilichia sp. y Anaplama sp. se presenta en la tabla 3. Unicamente se encontró diferencias entre las medias de la concentración de plaquetas, siendo los animales positivos los que presentaron una media menor.

\section{DISCUSIÓN}

Se detectó Anaplasma sp. y Ehrlichia sp. en perros con signos clínicos sospechosos de enfermedad, de los distritos de Huánuco, Amarilis y Pilcomarca. Es la primera vez que se detecta Anaplasma en Huánuco, y también la coinfección de Anaplasma sp.+ Ehrlichia sp.; en tanto que ya se había detectado y reportado la presencia de Ehrlichia sp. en esta provincia, y existían indicios de la presencia de Anaplasma; sin embargo, no se han reportado estudios similares que reporten a este último (Huerto y Dámaso, 2015; Tasayco et al., 2017).

En general, se ha sugerido que la coinfección de dos o más enfermedades trasmitidas por vectores, tal 
Tabla 2. Signos clínicos presentes con resultados resultados positivos y negativos a Ehrilichia sp. y Anaplama sp. en perros atendidos en consultorios veterinarios de Huánuco - Perú, 2020.

\begin{tabular}{|c|c|c|c|c|c|c|c|c|c|c|c|}
\hline \multirow{3}{*}{$\begin{array}{l}\text { Signo } \\
\text { clínico }\end{array}$} & \multirow{3}{*}{ Total } & \multicolumn{5}{|c|}{ Ehrlichia sp. } & \multicolumn{5}{|c|}{ Anaplasma sp. } \\
\hline & & \multicolumn{2}{|c|}{ Positivo } & \multicolumn{2}{|c|}{ Negativo } & \multirow{2}{*}{ Sig. } & \multicolumn{2}{|c|}{ Positivo } & \multicolumn{2}{|c|}{ Negativo } & \multirow{2}{*}{ Sig. } \\
\hline & & $\mathbf{n}$ & $\%$ & $\mathbf{n}$ & $\%$ & & $\mathbf{n}$ & $\%$ & $\mathbf{n}$ & $\%$ & \\
\hline \multicolumn{12}{|l|}{ Fiebre } \\
\hline $\mathrm{Si}$ & 47 & 41 & 87,2 & 6 & 12,8 & \multirow{2}{*}{0,556} & 32 & 68,1 & 15 & 31,9 & \multirow{2}{*}{0,171} \\
\hline No & 53 & 44 & 83,0 & 9 & 17,0 & & 29 & 54,7 & 24 & 45,3 & \\
\hline \multicolumn{12}{|c|}{ Mucosas pálidas } \\
\hline $\mathrm{Si}$ & 42 & 40 & 95,2 & 2 & 4,8 & \multirow{2}{*}{0,015} & 26 & 61,9 & 16 & 38,1 & \multirow{2}{*}{0,875} \\
\hline No & 58 & 45 & 77,6 & 13 & 22,4 & & 35 & 60,3 & 23 & 39,7 & \\
\hline \multicolumn{12}{|l|}{ Letargo } \\
\hline $\mathrm{Si}$ & 37 & 33 & 89,2 & 4 & 10,8 & \multirow{2}{*}{0,369} & 20 & 54,1 & 17 & 45,9 & \multirow{2}{*}{0,275} \\
\hline No & 63 & 52 & 82,5 & 11 & 17,5 & & 41 & 65,1 & 22 & 34,9 & \\
\hline \multicolumn{12}{|l|}{ Anorexia } \\
\hline $\mathrm{Si}$ & 30 & 25 & 83,3 & 5 & 16,7 & \multirow{2}{*}{0,760} & 18 & 60,0 & 12 & 40,0 & \multirow{2}{*}{0,893} \\
\hline No & 70 & 60 & 85,7 & 10 & 14,3 & & 43 & 61,4 & 27 & 38,6 & \\
\hline \multicolumn{12}{|c|}{ Hemorrágia } \\
\hline $\mathrm{Si}$ & 14 & 13 & 92,9 & 1 & 7,1 & \multirow{2}{*}{0,375} & 9 & 64,3 & 5 & 35,7 & \multirow{2}{*}{0,786} \\
\hline No & 86 & 72 & 83,7 & 14 & 16,3 & & 52 & 60,5 & 34 & 39,5 & \\
\hline \multicolumn{12}{|c|}{ Signos neurológico } \\
\hline $\mathrm{Si}$ & 11 & 9 & 81,8 & 2 & 18,2 & \multirow{2}{*}{0,754} & 8 & 72,7 & 3 & 27,3 & \multirow{2}{*}{0,398} \\
\hline No & 89 & 76 & 85,4 & 13 & 14,6 & & 53 & 59,6 & 36 & 40,4 & \\
\hline \multicolumn{12}{|l|}{ Ictericia } \\
\hline $\mathrm{Si}$ & 6 & 6 & 100 & 0 & 0,0 & \multirow{2}{*}{0,289} & 4 & 66,7 & 2 & 33,3 & 0.769 \\
\hline No & 94 & 79 & 84,0 & 15 & 16,0 & & 57 & 60,6 & 37 & 39,4 & $0, / 69$ \\
\hline Queratitis & & & & & & & & & & & \\
\hline $\mathrm{Si}$ & 1 & 1 & 100 & 0 & 0,0 & 0673 & 1 & 100 & 0 & 0,0 & 042 \\
\hline No & 99 & 84 & 84,8 & 15 & 15,2 & $0,0 / 3$ & 60 & 60,6 & 39 & 39,4 & $0,4<2$ \\
\hline
\end{tabular}

como se muestra en este estudio, podrían conducir a efectos inmunológicos más complejos, dificultando aún más el diagnóstico de estas asociaciones $\mathrm{y}$ posterior tratamiento (McCown et al., 2015).

Por otro lado, el 94.4\% de los perros de raza definida, también llamados "perros de raza", incluidos en el estudio, fueron positivos a Ehrlichia. Esta asociación es similar a lo demostrado en varios estudios que indican que los perros de raza son más susceptibles a la infección. Lo mismo no sucede con Anaplasma, tampoco en los casos de coinfección Anaplasma sp.+ Ehrlichia sp., donde no se logró demostrar asociación con la raza de los perros (Ybañez et al., 2018; Hoyos et al., 2007).
El sexo, el tamaño del pelaje, la presencia de garrapatas y el estilo de vida, no se encontraban asociados a la infección por Ehrlichia sp y Anaplasma sp. Sin embargo, en relación a la edad, los resultados indican mayor frecuencia en perros menores de 1 año $(73,3 \%)$, valores que son diferentes a otros estudios que reportan mayor frecuencia de Anaplasma sp. en perros mayores de 2 años (63,3\%) (Delgado y Montoya, 2018; Movilla et al., 2016), mayor frecuencia de Ehrlichia en perros mayores de 3 años (González et al., 2013; Movilla et al., 2016); y son similares a estudios que encuentran mayor frecuencia en perros hasta 1 año de edad (Paico, 2018), y una frecuencia de 100\% en perros menores de 2 años (Ortiz, 2012). También son diferentes en relación al estilo de vida, ya que algunos 
Tabla 3. Valores hematológicos con resultados positivos y negativos a Ehrilichia sp. y Anaplama sp. en perros atendidos en consultorios veterinarios de Huánuco - Perú, 2020.

\begin{tabular}{|c|c|c|c|c|c|c|c|c|}
\hline \multirow{2}{*}{$\begin{array}{c}\text { Párametro } \\
\text { hematologíco }\end{array}$} & \multicolumn{4}{|c|}{ Ehrlichia sp. } & \multicolumn{4}{|c|}{ Anaplasma sp. } \\
\hline & Nro. & Media & $\begin{array}{l}\text { Desviación } \\
\text { estándar }\end{array}$ & Sig. & Nro. & Media & $\begin{array}{l}\text { Desviación } \\
\text { estándar }\end{array}$ & Sig. \\
\hline \multicolumn{9}{|l|}{ Leucocito $\left(\times 10^{9} / \mathrm{L}\right)$} \\
\hline Negativo & 15 & 13,40 & 46,55 & \multirow{2}{*}{0,280} & 39 & 11,91 & 65,19 & \multirow{2}{*}{0,708} \\
\hline Positivo & 85 & 11,25 & 73,82 & & 61 & 11,36 & 74,35 & \\
\hline \multicolumn{9}{|l|}{ Eritrocito $\left(\times 10^{12} / \mathrm{L}\right)$} \\
\hline Negativo & 15 & 48,71 & 139,97 & \multirow{2}{*}{0,056} & 39 & 45,36 & 151,59 & \multirow{2}{*}{0,100} \\
\hline Positivo & 85 & 41,51 & 131,70 & & 61 & 40,82 & 120,77 & \\
\hline \multicolumn{9}{|c|}{ Hemoglobina (g/dL) } \\
\hline Negativo & 15 & 9,08 & 28,35 & \multirow{2}{*}{0,088} & 39 & 8,38 & 31,03 & \multirow{2}{*}{0,221} \\
\hline Positivo & 85 & 7,75 & 27,37 & & 61 & 7,68 & 25,40 & \\
\hline \multicolumn{9}{|l|}{ Hematocrito (\%) } \\
\hline Negativo & 15 & 33,61 & 109,68 & \multirow{2}{*}{0,086} & 39 & 30,94 & 112,03 & \multirow{2}{*}{0,260} \\
\hline Positivo & 85 & 28,81 & 96,79 & & 61 & 28,63 & 90,84 & \\
\hline \multicolumn{9}{|l|}{ VCM (fL) } \\
\hline Negativo & 15 & 70,35 & 39,01 & \multirow{2}{*}{0,125} & 39 & 68,65 & 50,22 & \multirow{2}{*}{0,846} \\
\hline Positivo & 85 & 68,48 & 43,97 & & 61 & 68,83 & 39,21 & \\
\hline \multicolumn{9}{|l|}{$\mathrm{HCM}(\mathrm{pg})$} \\
\hline Negativo & 15 & 18,54 & 12,84 & \multirow{2}{*}{0,872} & 39 & 18,20 & 22,66 & \multirow{2}{*}{0,260} \\
\hline Positivo & 85 & 18,69 & 35,25 & & 61 & 18,96 & 37,81 & \\
\hline \multicolumn{9}{|l|}{$\mathrm{CHCM}(\mathrm{g} / \mathrm{dL})$} \\
\hline Negativo & 15 & 26,34 & 11,59 & \multirow{2}{*}{0,370} & 39 & 26,54 & 21,31 & \multirow{2}{*}{0,263} \\
\hline Positivo & 85 & 27,02 & 28,70 & & 61 & 27,16 & 29,86 & \\
\hline \multicolumn{9}{|l|}{ Plaquetas $\left(\times 10^{9} / \mathrm{L}\right)$} \\
\hline Negativo & 15 & 177,93 & 113,11 & \multirow{2}{*}{0,017} & 39 & 124,41 & 89,77 & \multirow{2}{*}{0,580} \\
\hline Positivo & 85 & 106,13 & 103,96 & & 61.00 & 112,10 & 118,52 & \\
\hline
\end{tabular}

reportan una alta prevalencia en perros que viven fuera de la casa en forma libre y aquellos infestados con garrapatas (Movilla et al., 2016; Scorpio et al., 2008). El signo clínico de mucosas pálidas está asociado con la infección por Ehrlichia sp., pero no con Anaplasma sp.; los estudios reportan que los signos clínicos son muy variados (Harrus y Waner, 2011; Ybañez et al., 2018). Asimismo, los signos clínicos de fiebre, anorexia, letargia, ictericia, hemorragia, signos neurológicos y queratitis; no se asocian a Ehrlichia sp. y Anaplasma sp. Este resultado se explica por que en ambas enfermedades se presenta una disminución de los valores hematológicos en los perros, principalmente eritrocitos, hemoglobina y hematocrito; y es diferente a otros reportes que encuentran una alta frecuencia de signos clínicos que están asociados estadísticamente a ambos gérmenes (René-Martellet et al., 2015).
El valor hematológico asociado con Ehrlichiosis es el número de plaquetas, que en esta enfermedad estaba disminuído. Los valores referenciales de plaquetas en perros se encuentran entre $150,000-500,000 \mathrm{x}$ $\mu 1$ (Bossa-Miranda et al., 2012), con una media de 300,000 . En el estudio realizado, el promedio estaba en 106,130, con valores tan bajos como de 8,000 (González et al., 2013; Harrus et al., 1996). Esta trombocitopenia que se presentó en los perros del estudio, es similar a muchos reportes que indican una marcada trombocitopenia al inicio de la infección (Bulla et al., 2004; Harrus et al., 1997; Gaunt et al., 2010).

Asimismo, el número promedio de eritrocitos fue 4'151,000 x L, de hemoglobina $7.75 \mathrm{~g} \times \mathrm{dl}$, 
y de hematocrito 28.81\%; interpretandose como Anemia, ya que sus valores referenciales son: $5^{\prime} 000,000-8^{\prime} 000,000$ x L, $12-18 \mathrm{~g}$ x dl, y $37-$ $55 \%$ respectivamente (Hoyos et al., 2007; Paniagua y Guzmán, 2010; Romero et al., 2011).

Este estudio enfatiza la importancia del monitoreo de las enfermedades trasmitidas por vectores, con el propósito de determinar la frecuencia de estas enfermedades, el desarrollo y evaluación de riesgos, así como la implementación de una medicina preventiva y otros medios de control y prevención. Los resultados demuestran la necesidad de supervisar continuamente los lugares endemicos en donde los animales se encuentran mas expuestos a estas enfermedades.

\section{Correspondencia:}

Walter Richard Tasayco Alcantara

Correo electrónico: rtasayco@unheval.edu.pe

\section{REFERENCIAS BIBLIOGRAFICAS}

1. Alho, A. M., Lima, C., Latrofa, M. S., Colella, V., Ravagnan, S., Capelli, G., ... \& Otranto, D. (2017). Molecular detection of vector-borne pathogens in dogs and cats from Qatar. Parasites \& vectors, 10(1), $1-5$.

2. Barbosa, M. A. B., Peixoto, T. M. P., Fonseca, B. F., Silva, T. S., Freiria, L. M., Pereira, S. A. P., \& Dias, H. D. (2018). Hemoparasitos em caninos do município de Araguaína, Tocantins. Revista Brasileira de Higiene e Sanidade Animal: RBHSA, 12(4), 487-494.

3. Bossa-Miranda, M. A., del C Valencia-Celis, V., Carvajal-Giraldo, B. A., \& Ríos-Osorio, L. A. (2012). Valores de referencia del hemograma en perros sanos entre 1 y 6 años de edad, atendidos en el Hospital Veterinario-Universidad de Antioquia, 2002-2009. Revista Colombiana de Ciencias Pecuarias, 25(3), 409-416.

4. Bulla, C., Takahira, R. K., Araújo Jr, J. P., AparecidaTrinca, L., Lopes, R. S., \& Wiedmeyer, C. E. (2004). The relationship between the degree of thrombocytopenia and infection with Ehrlichia canis in an endemic area. Veterinary Research, 35(1), 141146.

5. Daniel, W. B. (2002). Base para el análisis de las ciencias de la salud. Editorial Limusa-Wiley.

6. Delgado, I. N., Montoya, A. M. (2018). Influencia de la edad y el sexo sobre la prevalencia de Anaplasma $s p$. en caninos (Canis familiaris) atendidos en Clinicas Veterinarias en los distritos de José Leonardo Ortiz, La Victoria y Chiclayo. Julio diciembre 2017. [Tesis pregrado]. Universidad Nacional Pedro Ruiz Gallo.

7. Ferreira da Silva, G., do Nascimento, A., Girotto, A.,
Taroda, A., Vidotto, M. C., Garcia, J. L., ... \& Vidotto, O. (2012). Occurrence of Ehrlichia canis and Anaplasma platys in household dogs from northern Parana. Revista Brasileira de Parasitologia Veterinária, 21, 379-385.

8. Gaunt, S. D., Beall, M. J., Stillman, B. A., Lorentzen, L., Diniz, P. P. V. P., Chandrashekar, R., \& Breitschwerdt, E. B. (2010). Experimental infection and co-infection of dogs with Anaplasma platys and Ehrlichia canis: hematologic, serologic and molecular findings. Parasites \& vectors, 3(1), 33.

9. González A.M., Rojas E.F., Pulido-Medellín M.O., \& García-Corredor D.J. (2013). Correlación entre hemograma y frotis sanguíneo para determinar E. canis en la vereda Peñitas de Puente Nacional. Revista Ciencia y Agricultura, 10(1), 17-23

10. Greene, C. E. (2008). Enfermedades Infecciosas del perro y el gato, Cap 28 "Ehrlichiosis, neorickettsiosis, anaplasmosis e infección por Wolbachia”. Elsevier Health Sciences,

11. Harrus, S., Aroch, I., Lavy, E., \& Bark, H. (1997). Clinical manifestations of infectious canine cyclic thrombocytopenia. Veterinary Record, 141(10), 247250.

12. Harrus, S., Waner, T., Eldor, A., Zwang, E., \& Bark, H. (1996). Platelet dysfunction associated with experimental acute canine ehrlichiosis. Veterinary Record, 139(12), 290-293.

13. Harrus, S., \& Waner, T. (2011). Diagnosis of canine monocytotropic ehrlichiosis (Ehrlichia canis): an overview. The Veterinary Journal, 187(3), 292-296.

14. Hoyos, L., Li, O., Alvarado, A., Suárez, F., \& Díaz, D. (2007). Evaluación del examen hematológico en el diagnóstico de ehrlichiosis canina. Revista de Investigaciones Veterinarias del Perú, 18(2), 129135.

15. Huerto-Medina, E., \& Dámaso-Mata, B. (2015). Factores asociados a la infección por Ehrlichia canis en perros infestados con garrapatas en la ciudad de Huánuco, Perú. Revista Peruana de Medicina Experimental y Salud Pública, 32, 756760.

16. Instituto Nacional de Estadística e Informática (2017). Censos nacionales, XII de Población, VII de Vivienda y III de comunidades indígenas. Instituto Nacional de Estadística e Informática. www.censo2017.inei.gob. pe

17. Kelly, P. J., Xu, C., Lucas, H., Loftis, A., Abete, J., Zeoli, F., ... \& Wang, C. (2013). Ehrlichiosis, babesiosis, anaplasmosis and hepatozoonosis in dogs from St. Kitts, West Indies. PLoS One, 8(1), e53450.

18. McCown, M. E., Monterroso, V. H., \& Cardona, W. (2015). Monitoreo de Ehrlichia canis, Anaplasma phagocytophilum, Borreliaburgdorferi, y Dirofilaria immitis en perros de tres ciudades en Colombia. CES Medicina Veterinaria y Zootecnia, 10(2), 224-231. 
19. Movilla, R., García, C., Siebert, S., \& Roura, X. (2016). Countrywide serological evaluation of canine prevalence for Anaplasma spp., Borrelia burgdorferi (sensu lato), Dirofilaria immitis and Ehrlichia canis in Mexico. Parasites \& vectors, 9(1), 1-12.

20. Ortiz, M. (2012). Seroprevalencia de Ehrli-chia canis y Anaplasma phagocytophium en caninos vagabundos de dos sectores urbanos de la ciudad de Talca, Región del Maule. [Tesis Pregrado]. Universidad Santo Tomás.

21. Paico S.C.M. (2018). Prevalencia de Anaplasmosis en caninos atendidos en la Clínica Veterinaria Pet's Park, La Victoria, Septiembre 2016 - Septiembre 2017. [Tesis pregrado]. Universidad Nacional Pedro Ruiz Gallo.

22. Paniagua, M.L.R., \& Guzmán, C.J. (2010). Características hematológicas, bioquímicas e histopatológicas de ehrlichiosis canina. [Tesis pregrado]. Universidad Autónoma Gabriel René Moreno.

23. René-Martellet, M., Lebert, I., Chêne, J., Massot, R., Leon, M., Leal, A., ...\& Halos, L. (2015). Diagnosis and incidence risk of clinical canine monocytic ehrlichiosis under field conditions in Southern Europe. Parasites \& vectors, $8(1), 1-10$.

24. Romero, B., Padilla, A., \& Alvarado, E. (2011). Cambios hematologicos en pacientes positivos a ehrlichiosis canina en la ciudad de Lázaro Cárdenas Michoacán. [Tesis pregrado]. Universidad Michoacana de San Nicolás de Hidalgo.

25. Scorpio, D. G., Wachtman, L. M., Tunin, R. S., Barat, N. C., Garyu, J. W., \& Dumler, J. S. (2008). Retrospective clinical and molecular analysis of conditioned laboratory dogs (Canis familiaris) with serologic reactions to Ehrlichia canis, Borrelia burgdorferi, and Rickettsia rickettsii. Journal of the American Association for Laboratory Animal
Science, 47(5), 23-28.

26. Simpson, R. M., Gaunt, S. D., Hair, J. A., Kocan, K. M., Henk, W. G., \& Casey, H. W. (1991). Evaluation of Rhipicephalus sanguineus as a potential biologic vector of Ehrlichia platys. American journal of veterinary research, 52(9), 1537-1541.

27. Stich, R. W., Schaefer, J. J., Bremer, W. G., Needham, G. R., \& Jittapalapong, S. (2008). Host surveys, ixodid tick biology and transmission scenarios as related to the tick-borne pathogen, Ehrlichia canis. Veterinary parasitology, 158(4), 256-273.

28. Tasayco, W., Carrión, F., Apac, A. (2017). Reporte de un caso clínico de ehrlichiosis canina en la ciudad de Huánuco, Perú. REDVET, 18(12), 1-7

29. Waner, T., Harrus, S., Jongejan, F., Bark, H., Keysary, A., \& Cornelissen, A. W. (2001). Significance of serological testing for ehrlichial diseases in dogs with special emphasis on the diagnosis of canine monocytic ehrlichiosis caused by Ehrlichia canis. Veterinary parasitology, 95(1), 1-15.

30. Ybañez, A. P., Perez, Z. O., Gabotero, S. R., Yandug, R. T., Kotaro, M., \& Inokuma, H. (2012). First molecular detection of Ehrlichia canis and Anaplasma platys in ticks from dogs in Cebu, Philippines. Ticks and Tick-borne diseases, 3(5-6), 288-293.

31. Ybañez, R. H. D., Ybañez, A. P., Arnado, L. L. A., Belarmino, L. M. P., Malingin, K. G. F., Cabilete, P. B. C., ... \& Xuan, X. (2018). Detection of Ehrlichia, Anaplasma, and Babesia spp. in dogs in Cebu, Philippines. Veterinary World, 11 (1): 14-19.

32. Zhang, J., Liu, Q., Wang, D., Li, W., Beugnet, F., \& Zhou, J. (2017). Epidemiological survey of ticks and tick-borne pathogens in pet dogs in southeastern China. Parasite, 24, 35. https://doi. org/10.1051/parasite/2017036 